\title{
Analytical Assessment of Tooling Requirements for FMS Design and Operation
}

\author{
S. C. Silva
}

Universidade do Minho,

Campus de Gualtar, 4710 - Braga, Portugal, phone: $+351+53$

60 4455, fax:+351+5360 4456, email scarmo@ci.uminho.pt

\begin{abstract}
FMS's do not work well if the required amount of tools to carry out processing, during a given manufacturing period, are not available. High operating efficiency can be ensured, once the right number of tools of each type is available, by conveniently solving the problem of assigning tools and parts to machines. This has been identified as the FMS loading problem (Stecke, 1981). Moreover it is essential that good manufacturing control of part processing priority is realised.

In this paper an analytical method is presented for initial assessment of the total number of required tools for efficiently running an FMS. The method is based on tool life duration and on a concept referred to as tool cycle time, as well as on the planned manufacturing period. This is usually taken as the time frame for the solution of the FMS loading problem.

Two fundamental situations are considered, namely that of static tool loading and dynamic tool loading.
\end{abstract}

\section{Keywords}

Flexible manufacturing system, static tool loading, dynamic tool loading.

\section{INTRODUCTION}

In simplified terms a Flexible Manufacturing System, FMS, may be defined as a computer aided manufacturing system able to simultaneously manufacture a variety of parts through computer control of processing and material flow. 
Much research has been carried out to solve the FMS loading problem identified by Stecke (1981). The problem and a few variations of it, for a number of different constraints, have been addressed by Sodhi 1994, Sarin 1987, Rajagopalan 1986, Stecke 1983 and other authors. In most cases considerable simplification of reality is assumed.

The development of a FMS, requires technical, economical and performance analysis of the alternatives for control, machining and material flow systems, (Warnecke 1982, Eversheim 1980, Storr 1979).

A tool flow system, TFS, is an integral part of the material flow system within a FMS. TFS's deal with the storage, transport and handling of tools which are exchanged at the machine spindles, and can be configured in many different ways (Silva, 1997). Therefore tool flow systems are conceived to handle a set of important manufacturing aids, i.e., the machining tools to be used by the machines to carry out the processing of parts.

This paper addresses the analytical estimation, of the number of tools to be loaded in the machines to run an FMS.

Two fundamental situations are considered, namely that of static tool loading and dynamic tool loading. These situations are closely related to the concepts of static tool change and dynamic tool change presented by Sodhi (1994). In static tool loading tools are only loaded at the start of each planned manufacturing period in such a way that a complete tooling autonomy exists during that period. Here no tool recycling is allowed In dynamic tool loading tools can be removed from, and fed into, the system during the planned manufacturing period.

The analytical methodology used can easily be extended to the determination of other manufacturing aids in FMS or other manufacturing and assembly systems.

\section{NUMBER OF TOOLS TO RUN A FMS}

\subsection{Number of tools under static tool loading}

While tool variety is primarily determined by the variety of part operations, the total number of tools required is dependent on the length of the manufacturing period for which tooling autonomy is desired, figure 1 . Under static tool loading tools are loaded only at the beginning of each planned manufacturing period.

\section{Tools with identical tool lives}

The number, $f$, of tools required to operate a single machine during a single time unit is given by:

$$
f=1 / t_{l}
$$

$t_{l}$ is the tool life time.

Any fraction of tool, should be interpreted as a tool which is required only for an equivalent fraction of tool life duration.

Under static tool loading having to run an FMS with a number of machines $m$ for an autonomous period of duration $T$ the total number of tools, $F$, required is given by: 


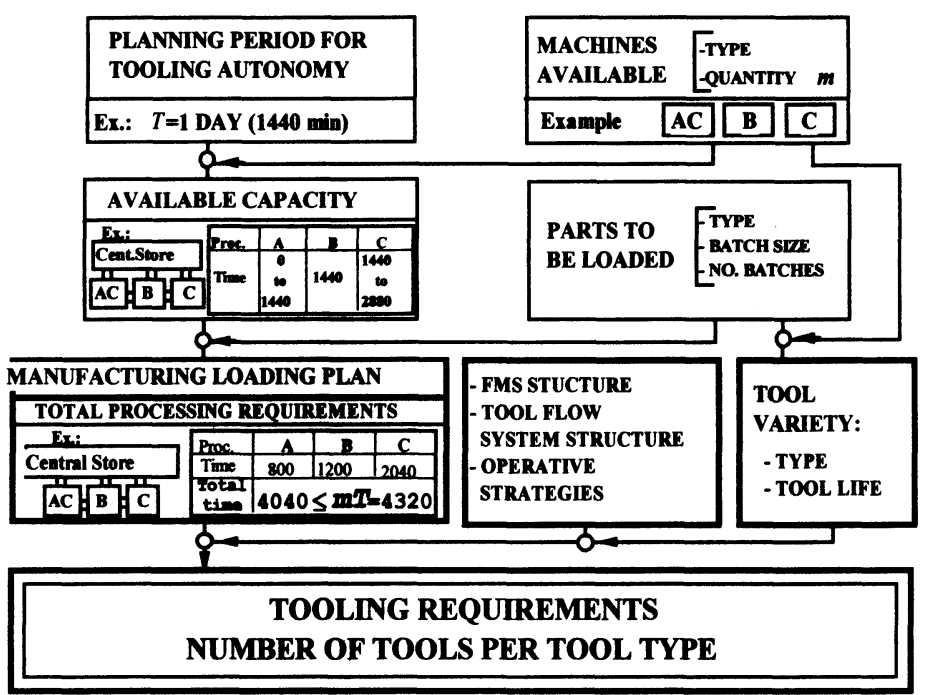

Figure 1 Tool requirements for a manufacturing planned period

$$
F=m T f
$$

or

$$
F=m T \frac{1}{t_{l}}
$$

As an example, considering a manufacturing period of a 3 eight hour shifts, i.e., a 24 hour day work, and 10 machines in the system, for tools with tool life of 20 minutes, applying equation ( 3 '), the number of tools required is:

$$
F=10 * 24 * 60 * \frac{1}{20}
$$

$F=720$ tools

Therefore, for complete tooling autonomy, during the 24 hour manufacturing period, a total of 720 tools would be necessary. The result assumes that machines are fully and constantly utilised in actual machining during the planned manufacturing period $T$ and that all tools are used to their full lives.

When expected average machine utilisation in actual machining is $U$, this would mean that the machining time per machine is $U$ times $T$. On the other hand, due to technical, organisational or economical reasons resulting from system operation, usually tool life is not used up completely. Only a percentage, say $\beta$, of tool life duration is used. In this case, a 
generic expression for the number $F$ of required tools, which also takes into account the average machine utilisation, can be written as:

$$
F=U m T \frac{1}{\beta t_{l}}
$$

\section{Taking account of tool life differences}

When more than a single type of tool, with different tool lives, are going to be used, the number $\bar{f}$ of tools required, to continuously operate a machine during a single unit time, is the average number of tools, over all tool types, and is given by:

$$
\bar{f}=\frac{1}{n} \sum_{i=1}^{n} f_{i}
$$

- $\bar{f}$ is the number of tools that, on average, will be necessary for running a machine during a single time unit;

- $n$ is the number of different tools to be used;

- $f_{i}$ is the number of tools of type $i$ which would be required to operate continuously a machine, during a single time unit, and is given by: $f_{i}=1 / t_{l_{i}}$.

Therefore, equation (4) can be rewritten as:

$$
\bar{f}=\frac{1}{n} \sum_{i=1}^{n} \frac{1}{t_{l_{i}}}
$$

which allows, to write the average number, $\bar{F}$, of tools necessary to run an FMS with $m$ machines for an autonomous period $T$, as:

$$
\bar{F}=m T \frac{1}{n} \sum_{i=1}^{n} \frac{1}{t_{l_{i}}}
$$

and, generalising for taking average machine utilisation $U$ into account and the proportion $\beta$ of tool life used, it will become:

$$
\bar{F}=U m T \frac{1}{n} \sum_{i=1}^{n} \frac{1}{\beta t_{l_{i}}}
$$

- $\bar{F}$ is the average number of required tools for running a FMS under static tool loading;

- $m$ is the number of machines in the FMS;

- $T$ is length of the manufacturing planned period under which tooling autonomy exists;

- $n$ is the number of different tools to be used; 
- $\beta$ is the percentage of used tool life;

- $t_{l_{i}}$ is the tool life of tool type $i$.

\subsection{Number of required tools to run an FMS under dynamic tool loading}

Dynamic tool loading is characterised by dynamic tool changing (Sodhi, 1994), i.e. the possibility of dynamically changing tools at the magazines of the machines, and, also, feeding the manufacturing system with new tools to replace those which wear out and are removed, during the planned working period. This means that full tool recycling in adopted.

Under dynamic tool loading the number of required tools, at any time, to run a FMS, can be considerably reduced. To determine this number let us consider first that $t_{f}$ is the average tool flow time, inside the system, until tool life ends. This time will be called tool cycle time, figure 2. Tool cycle time includes not only the time the tool is involved in processing but also the time the tool is delayed in the system due to waiting, transport, handling and set-up or preparation.

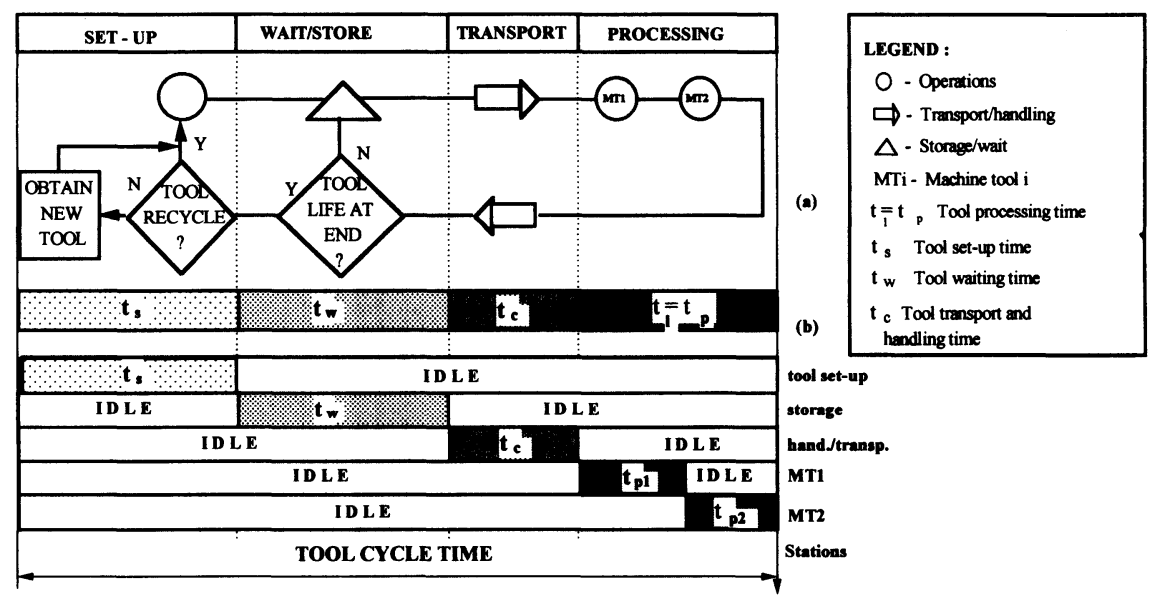

Figure 2 (a) - Schematic representation of the tool cycle time in an FMS

(b) - Multiple activity cycle diagram for a single tool.

After this time $t_{f}$ has elapsed a tool tip may be replaced and the tool reused. Alternatively, for non reusable tools, tool replacement is required. In either case the average number of tools in the system, at any time, is kept constant. It is, in fact, as if the tools were recycled with new tool lives.

A dummy usage rate, $R$, per tool can, therefore, be established as a function of the manufacturing planned period $T$ and average tool cycle time $t_{f}$.

This usage rate, $R$, is, by definition, given by

$R=\frac{T}{t_{f}}$ 
- $R$ can be interpreted as the number of times that, on average, each tool is reused or substituted after tool life ends during a planned manufacturing period, with first time use also included.

- $t_{f}$ is the average tool cycle time.

The number of required tools in a FMS at any time, under dynamic tool loading is, therefore, scaled down, in relation to the number of tools under static loading, by the $R$ factor.

\section{Tools with identical tool lives}

If tools are identical or have the same tool life duration, the number $F_{D}$ of required tools in a FMS, at any time, under dynamic tool loading, as referred above, can be obtained from scaling down the number of tools $F$ under static tool loading:

$$
F_{D}=\frac{F}{R}
$$

or, from equations (3') and (7):

$$
F_{D}=\left(m \frac{T}{t_{l}}\right) /\left(\frac{T}{t_{f}}\right)
$$

and therefore:

$$
F_{D}=m \frac{t_{f}}{t_{l}}
$$

Equation (9) shows that the number of tools required in a FMS, at any time, is not dependent on the length of the manufacturing period, $T$, but solely dependent on tool cycle time $t_{f}$, on tool life, $t_{l}$, and on the number of machines, $m$ in the system. Moreover we can conclude that, on average, for each machine in the system a number $t_{f} / t_{l}$ of tools is required. As figure 2 suggests, $t_{f}$ can be expressed as:

$t_{f}=t_{l}+t_{s}+t_{c}+t_{w}$

- $t_{l}$ is the tool life time ;

- $t_{s}$ is the tool set-up time per tool cycle;

- $t_{c}$ is the tool transport and handling times per tool cycle;

- $t_{w}$ is the tool waiting time, due to storage and buffering during manufacturing, per tool cycle.

Therefore $F_{D}$ can be rewritten as: 
$F_{D}=m \frac{\left(t_{l}+t_{s}+t_{c}+t_{w}\right)}{t_{l}}$

or

$$
F_{D}=m+m\left(\frac{t_{s}}{t_{l}}\right)+m\left(\frac{t_{c}}{t_{l}}\right)+m\left(\frac{t_{w}}{t_{l}}\right)
$$

or

$$
F_{D}=F_{m}+F_{s}+F_{c}+F_{w}
$$

- $\quad F_{m}$ are the tools used at machining, given by $\mathrm{m}$;

- $F_{s}$ are the tools used at preparation and set-up, given by $m\left(t_{s} / t\right)$;

- $F_{c}$ are the tools being carried or handled, given by $m\left(t_{c} / t\right)$;

- $F_{w}$ are the tools waiting in system, given by $m\left(t_{w} / t\right)$;

From equation (12) we can see that reducing tool waiting and the time of some activities such as transport and tool set-up can mean a reduction in tools required in the system. Dependent upon the amount of time, this could be a substantial reduction. In the extreme, the minimum number of tools is equal to one per machine and corresponds to the permanent use of a single tool at each machine.

Equation (11), for the number $F_{D}$ of required tools in a FMS, can be adjusted to take account of tool replacement before tool life ends, with $\beta$ as the percentage of used tool life:

$$
F_{D}=m \frac{\left(\beta t_{l}+t_{s}+t_{c}+t_{w}\right)}{\beta t_{l}}
$$

\section{Minimum number of required tools in an FMS}

The minimum number $F_{D_{\min }}$ of tools to run a FMS is obtained when tools do not wait, that is, they are constantly used for part processing, being transported or set-up. In this case:

$$
F_{D_{\min }}=m \frac{\left(\beta t_{l}+t_{s}+t_{c}\right)}{\beta t_{l}}
$$

This assumes that tools flow continuously between machines and tool preparation area and, therefore, theoretically, no tool stores are necessary. This tooling situation is similar to the flow of parts in a pure part flow line without intermediate storage buffers between stations. 
Tool requirements considering tool cycle time and tool life duration differences The number of required tools in the system has been determined considering that all tools in the system have identical tool lives and tool cycle times.

Taking account of equation (9) and differences on tool life and tool cycle times the average number $\bar{F}_{D}$ of required tools for running a FMS under dynamic tool loading is:

$$
\bar{F}_{D}=m \frac{1}{n} \sum_{i=1}^{n} \frac{t_{f_{i}}}{t_{l_{i}}}
$$

and, by decomposing $t_{f_{i}}$ in its elements and taking the percentage $\beta$ of tool life used, it will become:

$\bar{F}_{D}=m \frac{1}{n} \sum_{i=1}^{n} \frac{\left(\beta t_{l_{i}}+t_{s_{i}}+t_{c_{i}}+t_{w_{i}}\right)}{\beta t_{l_{i}}}$

- $\bar{F}_{D}$ is the average number of required tools for running a FMS under dynamic tool loading;

- $m$ is the number of machines in the FMS;

- $n$ is the number of different tools to be used;

- $\beta$ is the percentage of used tool life;

- $t_{l_{i}}$ is the tool life of tool type $i$;

- $t_{s_{i}}$ is the tool set-up time per tool cycle;

- $t_{c_{i}}$ is the tool transport and handling times per tool cycle;

- $t_{w_{i}}$ is the tool waiting time, due to storage and buffering during manufacturing, per tool cycle.

The equation (16) takes account of differences in individual tool life, tool set-up. tool transport and handling and tool waiting times. However, the waiting time, in the tool cycle time, can be seen as a safety factor which can be adjusted according to the tooling flow system response time, i.e., the readiness for tool set-up and tool transport and handling. Therefore, the waiting time is dependent on the strategy of FMS operation, but particularly dependent on the tool flow system configuration (Silva, 1997). Thus, for example, the existence of a single tool central store, dynamically feeding the machines of the FMS which have each a single position tool buffer for the tool to process the next operation, is likely to reduce the overall waiting time of tools with consequent reduction of the number of tools required, at any time, in the system.

\section{DISCUSSION AND CONCLUSIONS}

The method presented here is analytical and does not take into account the operation dynamics of FMS's. As a consequence, it offers values which can only be considered as a first 
approximation to the required number of tools necessary to operate a FMS. This number is determined under two situations, namely static tool loading and as dynamic tool loading.

The precise number of each tool required is highly dependent on many aspects of system flexibility. This is related to system design and dynamics of system operation. Such dynamics results, for example, from time variability of processing and handling as well as from operative strategies and sequencing priority rules. A rigorous approach to the determination of tools required for a particular FMS would, most probably, require the use of a detailed simulation model of the FMS, run every time a FMS loading solution was required.

The number of tools required cannot be smaller than the number of different tools necessary to process the selected parts in the FMS. This number is, therefore, a lower bound to the total number of tools necessary. Further, as seen, under static tool loading, the length of the manufacturing period is determinant of the amount of tools required. Under dynamic tool loading this period is not important.

Manufacturing systems which are running for some time could take particular advantage of the analytical assessment presented. In fact these systems are in a good position for providing the data necessary by the method. Thus an evaluation of shortage or excess of tooling resources could be carried out. Moreover, the method can be useful in FMS planning or replanning because, by establishing estimations of the total number of tools, it helps in the estimation of requirements for tool storage and handling within a FMS. This, is relevant for the design of the tool flow system. Further, the estimation of the number of tools can help to make an initial and rough evaluation of performance of running FMS's under static or dynamic tool loading. This evaluation would be based on the number of tools really available as compared to the number which is required, estimated by the method.

The points discussed show that the analytical method presented can help reengineering manufacturing systems towards better design and operation.

\section{REFERENCES}

Eversheim, W and Herrmann, P., (1980) "Technical and Economical Planning of Automated Manufacturing Systems." Proc. 4th Int. Conf. on Production Research, Tokyo.

Rajagopalan, S. (1986) "Formulation and heuristic solutions for parts grouping and tool loading in Flexible Manufacturing Systems." Procs. of the 2nd ORSA/TIMS Conf. on Flexible Manufacturing Systems, Elsevier.

Sarin, S. C. and Chen, C. S. (1987) "The machine loading and tool allocation problem in a flexible manufacturing system." International Journal of Production Research, 25, No 7, 1081-94.

Silva, S.C. (1997) "Strategies and Fundamental Structures for FMS Tool Flow Systems." accepted to the OE/IFIP/IEEE Int. Conf. on Integrated and Sustainable Industrial Production"

Sodhi, M. S., Askin, R. G. and Sen, S. 1994" Multiperiod tool and production assignment in flexible manufacturing systems". International Journal of Production Research, 32, No.6 1281-94.

Stecke, K E. (1983) "Formulation and solution of non-linear integer production planning problems of FMS". Management Science, 29, 273-88.

Stecke, K. E. (1981) "Production Planning Problems for Flexible Manufacturing Systems". $\mathrm{PhD}$ Thesis, Purdue University. 
Storr, A. (1979) Plannung und realisierung flexibler Fertigungssysteme. Wt Zeitschrift für industriell Fertigung 60 No.11.

Warnecke, H.J. and Vettin G. (1982) Technical Investment Planning of Flexible Manufacturing Systems. Journal of Manufacturing Systems, 1 No.1.

\section{BIOGRAPHY}

Dr. S. Carmo Silva is a member of the Departamento de Produção e Sistemas at the Universidade do Minho, in Braga, Portugal. At present Dr. Carmo Silva is the head of the Industrial Management and Systems group. He got his $\mathrm{PhD}$, in 1988, from Loughborough University and his MSc degree from UWIST in U.K, both in the area of Production Systems and Management. He is also graduated in Mechanical Engineering by Oporto University, Portugal. His main academic and research intestests fall into the general concept of Manufacturing Systems Engineering and, in particular into the area of design and operation of integrated manufacturing and assembly systems. 